Journal of Engineering and Applied Sciences 14 (13): 4597-4602, 2019

ISSN: 1816-949X

(C) Medwell Journals, 2019

\title{
A New Technique for Support Vector Machine Parameters Optimization Based on Modiefied PSO Algorithm
}

\author{
${ }^{1}$ Sabah Khudhair Abbas, ${ }^{2}$ Abdullah Aziz Laftah, ${ }^{3}$ Hasanein D. Rjeib \\ ${ }^{1}$ College of Imam Kadhim for Islamic Sciences University, Baghdad, Iraq \\ ${ }^{2}$ Department of Computer Science, Faculty of Education for Girls, \\ ${ }^{3}$ Department of Electronic and Communications Engineering, University of Kufa, Kufa, Iraq
}

\begin{abstract}
Support vector machine can determine the global finest solutions in many complicated problems and it is widely used for human face classification in the last years. Nevertheless, one of the main limitations of SVM is optimizing the training parameters, especially when SVM used in face recognition domains. Various methodologies are used to deal with this issue such as PSO, OPSO, AAPSO and AOPSO. Nevertheless, there is a room of advancements in this kind of optimization process. Lately, an improved version of PSO is developed which is called modified PSO. In this study, a new technique based on modified PSO, called (Modified PSO-SVM) is proposed to optimize SVM parameters. The proposed scheme utilizes modified PSO to seek the finest parameters of SVM two human face datasets: SCface, CASIAV5 and CMU Multi-PIE face datasets are used in the experiments. Then, a comparison is done with the PSO-SVM, OPSO-SVM and AOPSO-SVM and it showed promising results in terms of accuracy.
\end{abstract}

Key words: Biometric, optimization, face recognition, $\mathrm{PSO}$, SCface, experiments

\section{INTRODUCTION}

"Support Vector Machine (SVM)" is a machine learning approach and it is one of the famous classification methods. It deals with high dimensional problems and works efficiently using a minor number of training examples. It works on the mechanism of risk minimization, that leads to the "global optimum" solutions (Hasan et al., 2013). The SVM approach has been adopted in many areas such as "text categorization" Joachims (1998), medical diagnostics Raikwal and Saxena (2012), "credit risk analysis" Yu et al. (2008), "information extraction" Li et al. (2005), face recognition Abdullah et al. (2017), etc. Nonetheless, the selection of the training parameters in SVM is a big challenge due to its effect on the stability performance of SVM. Lately, the well-known artificial intelligence algorithm, the "Particle Swarm Optimization (PSO)" that is announced by Eberhart and Kennedy (1995) has been applied to optimize the SVM parameters. The PSO inspiration came from the social actions between folks same as the groups of fish or birds blocking. Recently, many studies have used PSO to optimize the training parameters of SVM, one of the earlier studies introduced by Wei et al. (2011) and used effectively in face recognition domain. However, this issue still an open issue and there is an area of advancements to optimize the parameters of SVM. Newly, a new approach called modified PSO is proposed by Khadhraoui et al. (2016) which is an enhancement of the standard PSO algorithm and it is applied powerfully with $2 \mathrm{D}$ face recognition. Therefore, this study proposes a new face recognition scheme based on the modified PSO algorithm and SVM.

Literature review: For face recognition applications, many attempts have been adopted various forms of PSO algorithm to select the optimal parameters of SVM. One of the recent studies that applied the standard PSO with SVM was introduced by Wei et al. (2011). They used PCA algorithm for a feature extraction process and PSO utilized to find the best parameters of SVM. The FERET database is used in the experimental to evaluate their proposed PSO-SVM technique and the results shows that their PSO-SVM has higher recognition accuracy than the standard SVM and BPNN as well. Nevertheless, the standard PSO has a drawback which random population generation that might influence in the recognition result. Moreover, they adopted one database which is not enough to make sure that the PSO-SVM is reliable in the complex face recognition applications. Hasan et al. (2013) have been proposed a OPSO-SVM face recognition technique which used OPSO in order to solve the random generation of population problem. They utilized FERET and YALE face databases in order to examine the

Corresponding Author: Sabah Khudhair Abbas, College of Imam Kadhim for Islamic Sciences University, Baghdad, Iraq 
performance of OPSO-SVM and they compared it with conventional SVM and PSO-SVM methods. Their OPSO-SVM has achieved higher accuracy than the standard SVM and PSO-SVM. However, there is a limitation in OPSO which is the fixed value of velocity coefficients same as the standard PSO. In order to evade the problem of velocity coefficients in PSO, Abdulameer et al. (2014) were introduced AAPSO-SVM which is a combination of an "adaptive acceleration particle swarm optimization and support vector machine". They introduced a new mechanism to choose the velocity coefficients based on the fitness values of particles. They used YALE and CASIA face databases to evaluate the effectiveness of the AAPSO-SVM and they achieved promising results better than OPSO-SVM and PSO-SVM. Recently, Abdulameer et al. (2018) and his collages have proposed AOPSO-SVM which is a combination of OPSO-SVM and AAPSO-SVM in order to achieve high recognition results and they succeed to achieve higher recognition accuracy than over mentioned methods. So, that and in order to achieve higher recognition results, a new face recognition technique based on the recent developed method (modified PSO) and (SVM) is introduced in this study.

\section{MATERIALS AND METHODS}

Support vector machine classifier: A "Support Vector Machine (SVM)" is defined by a separating hyperplane and it is considered as discriminative classifier. It is a supervised learning which means if we given a "labeled training data", the algorithm can produce an optimum hyperplane that classifies the new examples. The hyperplane is a line separating a plane into two parts in 2D dimensional space Abdullah et al. (2017); Abdulameer et al. (2018). The classification procedure of SVM in brief is clarified in Fig. 1. The optimization problem for nonlinear decision surface is assumed as:

$$
\begin{aligned}
& \min \frac{1}{G, \zeta}\|G\|^{2}+P \sum_{i=1}^{n} \varsigma_{i} \\
& y_{i}\left(G . x_{i}+b_{i}\right) \geq 1-\zeta_{i}, \zeta_{i} \geq 0, i=1,2, \ldots, n
\end{aligned}
$$

where, $\mathrm{P}$ represents the penalty parameter which is a regularization constant that controls the "tradeoff" between the error minimization and margin maximization. So, the decision function of classification becomes:

$$
f(x)=\sin \left(\sum_{i=1}^{n} h_{i} y_{i} k e\left(x_{i}, x_{j}\right)+b_{i}\right)
$$

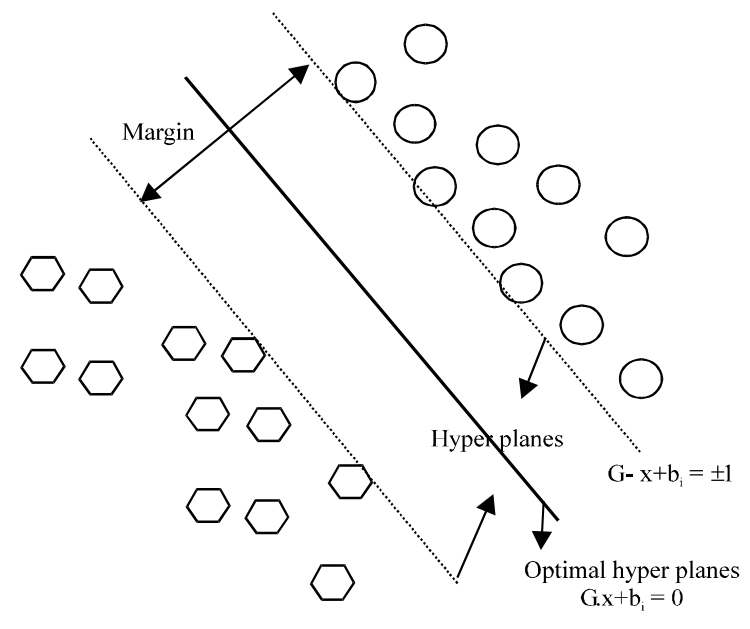

Fig. 1: The SVM classification procedure

In the previous formula, $h_{i}$ is the "Lagrange multipliers" and the kernel function is represented by $\operatorname{ke}\left(\mathrm{x}_{\mathrm{i}}, \mathrm{x}_{\mathrm{j}}\right)=\phi \mathrm{x}_{\mathrm{i}}, \phi \mathrm{x}_{\mathrm{j}}$. The goal of kernel function is to map the data in to "higher dimensional space" through using "nonlinear mapping" functions $\phi x$. The RBF which is "Radial basis function" is widely used in literature and we used it in SVM creation. It is defined by $\exp \left(-\left\|\mathrm{x}_{\mathrm{i}}-\mathrm{x}_{\mathrm{j}}\right\| / 2 \sigma^{2}\right), \sigma$ means a positive real number.

Particle swarm optimization: The PSO algorithm that was presented by Eberhart and Kennedy (1995) which is able to solve the problems that has continuous variables. In PSO algorithm, every particle discover the "solution space" seeking for an optimal solution. Kennedy (2011), Hussein and Habeeb (2018). The velocities and positions of the particles are updated according to the following Eq. 3 and 4:

$$
\begin{gathered}
\mathrm{Ve}_{\mathrm{i}}^{\mathrm{t}+1}=\mathrm{U}^{*} \mathrm{~V}_{\mathrm{i}}^{\mathrm{t}}+\mathrm{q}_{1} * \operatorname{ran}_{1} *\left(\mathrm{pe}_{\mathrm{ibest}}-\mathrm{A}_{\mathrm{i}}^{\mathrm{t}}\right)+ \\
\mathrm{q}_{2}^{*} \operatorname{ran}_{2} *\left(\mathrm{gl}_{\text {best }}-\mathrm{A}_{\mathrm{i}}^{\mathrm{t}}\right) \\
\mathrm{A}_{\mathrm{i}}^{\mathrm{t}+1}=\mathrm{A}_{\mathrm{i}}^{\mathrm{t}}+\mathrm{Ve}_{\mathrm{i}}^{\mathrm{t}+1}
\end{gathered}
$$

$\mathrm{L}$ is the size of the swarm and $\mathrm{I}=1,2, \ldots, \mathrm{L} . \mathrm{Ve}_{\mathrm{i}}$ denotes to the velocity of the current particle and $\mathrm{Ve}_{i}^{t+1}$ is new velocity of the particle; $U$ represents the inertia weight. $\mathrm{q}_{1}$ and $\mathrm{q}_{2}$ are two positive constants named as cognitive components, two independents random number in the range $[0,1]$ are represented by $\operatorname{ran}_{1}$ and $\operatorname{ran}_{2}, \mathrm{~A}_{1}^{t+1}$ refers to the position of the particle in the swarm, $p_{i \text {-best }}$ represents the finest gotten solution of the ith particle, $\mathrm{g} \mathrm{l}_{\text {best }}$ represents the finest solution of particle in the swarm: 


\section{RESULTS AND DISCUSSION}

The modified PSO: In order to improve the global search quality of the standard PSO in the modified PSO algorithm, the cognitive components $\left(\mathrm{q}_{1}, \mathrm{q}_{2}\right)$ and the inertia weight $\mathrm{U}$ have been formed (Khadhraoui et al., 2016). The updating on the velocity is represented as in the subsequent equation:

$$
\begin{aligned}
& \mathrm{Ve}_{\mathrm{i}}^{\mathrm{t}+1}=\mathrm{U}_{\text {new }} * \mathrm{~V}_{\mathrm{i}}^{\mathrm{t}}+\mathrm{q}_{1} * \operatorname{ran}_{1} *\left(\mathrm{pe}_{\text {ibest }}-\mathrm{A}_{\mathrm{i}}^{\mathrm{t}}\right)+ \\
& \mathrm{q}_{2} * \operatorname{ran}_{2} *\left(\mathrm{gl}_{\text {best }}-\mathrm{A}_{\mathrm{i}}^{\mathrm{t}}\right)
\end{aligned}
$$

Where:

$$
\mathrm{U}_{\text {new }}=\mathrm{U}_{\min }+\mathrm{U}^{*} \operatorname{ran}_{1}
$$

where, the U is represented as in the Eq. 7:

$$
\mathrm{U}=\mathrm{U}_{\mathrm{mac}}-\left[\left(\mathrm{U}_{\max }-\mathrm{U}_{\min }\right) * \text { iterc }_{\text {iter }}\right.
$$

Where:

$\mathrm{U}_{\min }=$ The minimum inertia weight

$\mathrm{U}_{\max }=$ The maximum inertia weight

$$
\begin{aligned}
& \mathrm{q}_{1}=\mathrm{q}_{1_{\max }}-\left(\mathrm{q}_{1_{\max }}-\mathrm{q}_{1 \min }\right) *\left(\frac{\text { iterc }}{\text { iter }_{\mathrm{m}}}\right) \\
& \mathrm{q}_{2}=\mathrm{q}_{2 \max }-\left(\mathrm{q}_{2 \max }-\mathrm{q}_{2 \min }\right) *\left(\frac{\text { iterc }}{\text { iter }_{\mathrm{m}}}\right)
\end{aligned}
$$

Where:

$\mathrm{q}_{1 \min }=$ The minimum, value cognitive components

$\mathrm{q}_{I_{\max }}=$ The maximum values of the cognitive component

$\mathrm{q}_{2 \min }=$ The minimum value of social component

$\mathrm{q}_{2 \max }=$ the maximum values of the social component

iterc $=$ Represents the current of iterations

iter $_{\mathrm{m}}=$ Represents the maximum values of iterations

For every particle in every generation process and in order to evaluate the velocity and the position for selecting the best search value, the fitness function is used. The fitness function able to provide the finest location of swarm space and the optimum "threshold" value. Hence, for every particle, the fitness function is evaluated. The particles develop by refreshing their speeds and positions dependent on their capacity to exploit the class detachment term demonstrated by the "scatter index" among the diverse classes. The means Mi of the equivalent classes and the mean M0 in the "feature space" are computed the following Eq. 10 and 11:

$$
D_{i}=1 / F_{i} \sum_{j=1}^{F_{i}} Z_{j}^{(i)}
$$

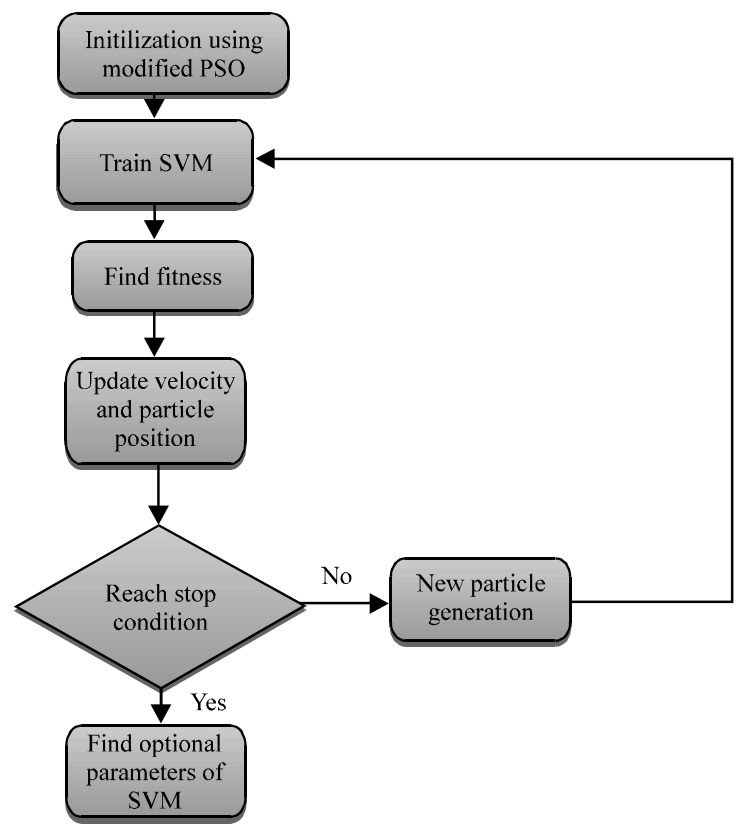

Fig. 2: The block diagram of the optimization using the modified PSO

$$
\mathrm{D}_{0}=1 / \mathrm{F} \sum_{\mathrm{i}=1}^{\mathrm{L}} \mathrm{F}_{\mathrm{i}} \mathrm{D}_{\mathrm{i}}
$$

where, $i=1,2, \ldots, L$ and $j=1,2, \ldots, F ; Z_{j}^{(1)}$ are the example images that belongs to class $Z_{i} ; F_{i}$ denotes to the number of images in every class; $D_{i}$ are the mean of equivalent classes and $D_{0}$ indicate to the mean in the feature space. The fitness function FIT is calculated by very simple Eq. 12 :

$$
\text { FIT }=\sqrt{\sum_{i=1}^{L}\left(D_{i}-D_{0}\right)^{t}\left(D_{i}-D_{0}\right)}
$$

Parameter optimization of SVM by the modified PSO: The SVM built by the "RBF kernel function" and it is based on $\mathrm{P}$ and $\sigma$ which are two user-determined parameters. At that time, the particle is involve $\mathrm{P}$ and $\sigma$. The process of the optimization process for SVM through the modified PSO is illustrated in Fig. 2 as follow:

Experimental exploration for the proposed face recognition technique: The whole procedure of the proposed face recognition technique based on the modified PSO-SVM is displayed in Fig. 3. After reading the face image, the LDA is adopted to extracts face features from the face image (Mosa, 2018). The resulted vector is used for training and testing the modified PSO-SVM Model.

We utilized three human face databases CMU Multi-PIE Gross et al. (2008), Anonymous (2007) and 


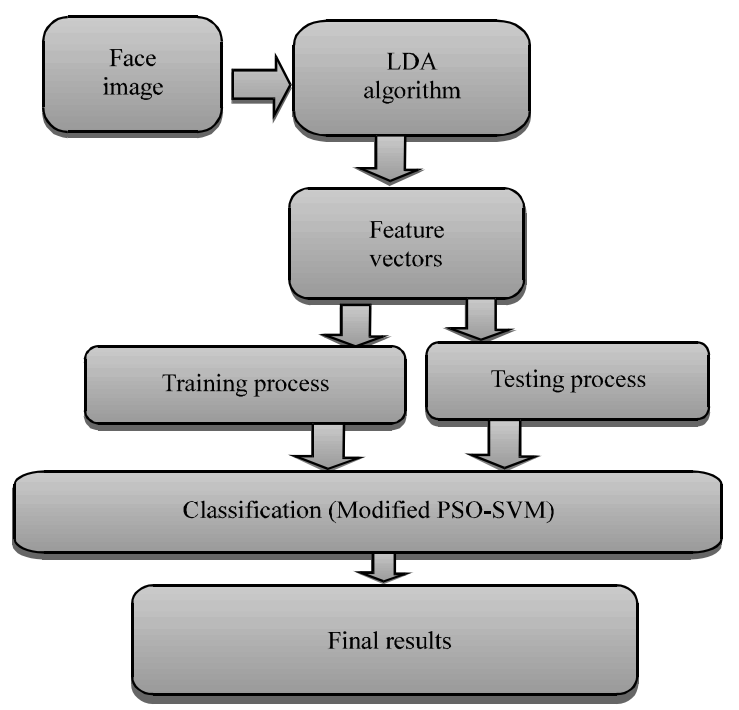

Fig. 3: The block diagram of the proposed face recognition technique
Scface face Grgic et al. (2011) dataset in order to test the performance of the proposed technique. The MATLAB environment is used for the experimentation. From every dataset, we chose 200 images for 50 subjects and the images were in different pose and illuminations. The images are divided in to $30 \%$ for training and $70 \%$ for testing. Figure 4 shows some of the samples images from each dataset.

The accuracy measurement is utilized to examine the performance of the proposed technique. In the experiments, images from different pose and illumination are adopted and ten rounds are adopted in the implementation. A comparison process for the proposed modified PSO-SVM, SVM, OPSO-SVM and AAPSO-SVM is employed in order to illustrate the efficiency of the proposed method. The experimental results shows that modified PSO-SVM has attained the highest accuracy among the other methods as shown in Table 1 and Fig. 5, respectively.

(a)

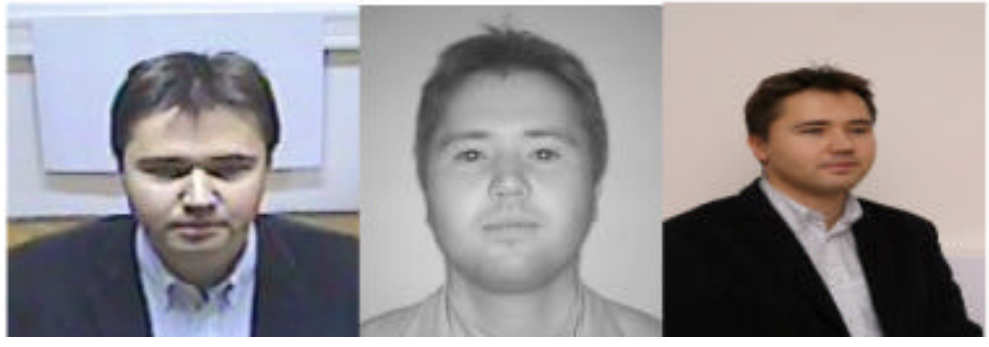

(b)
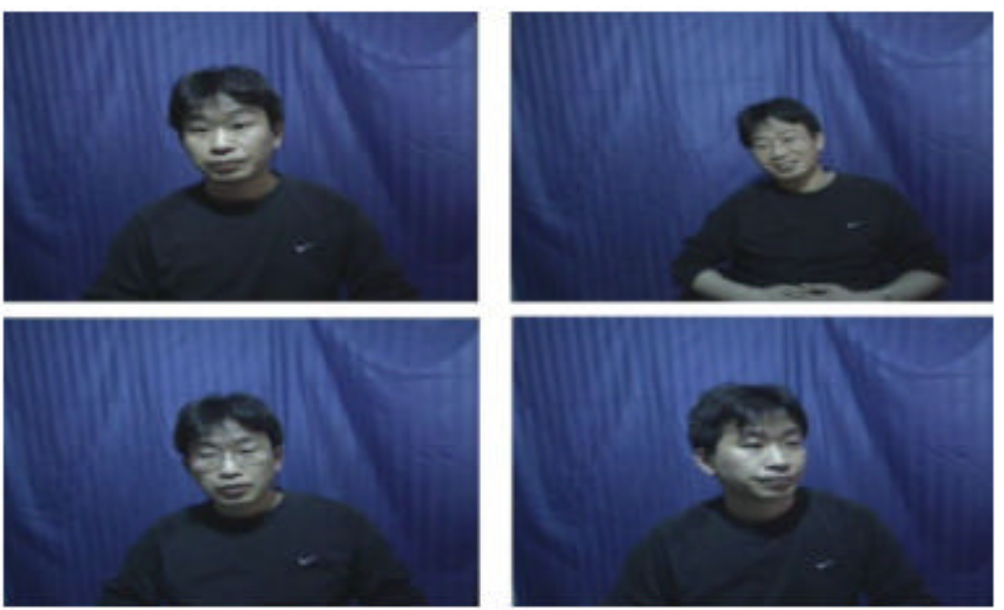

(c)

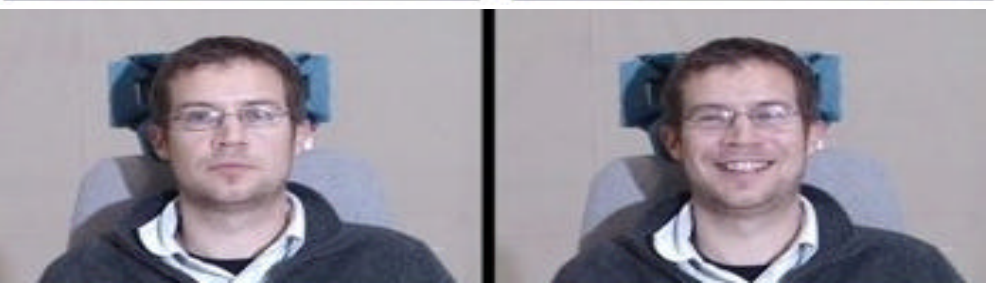

Fig. 4: Samples from the three datasets: a-c) SCface, CASIAV5 and CMU Multi-PIE dataset 


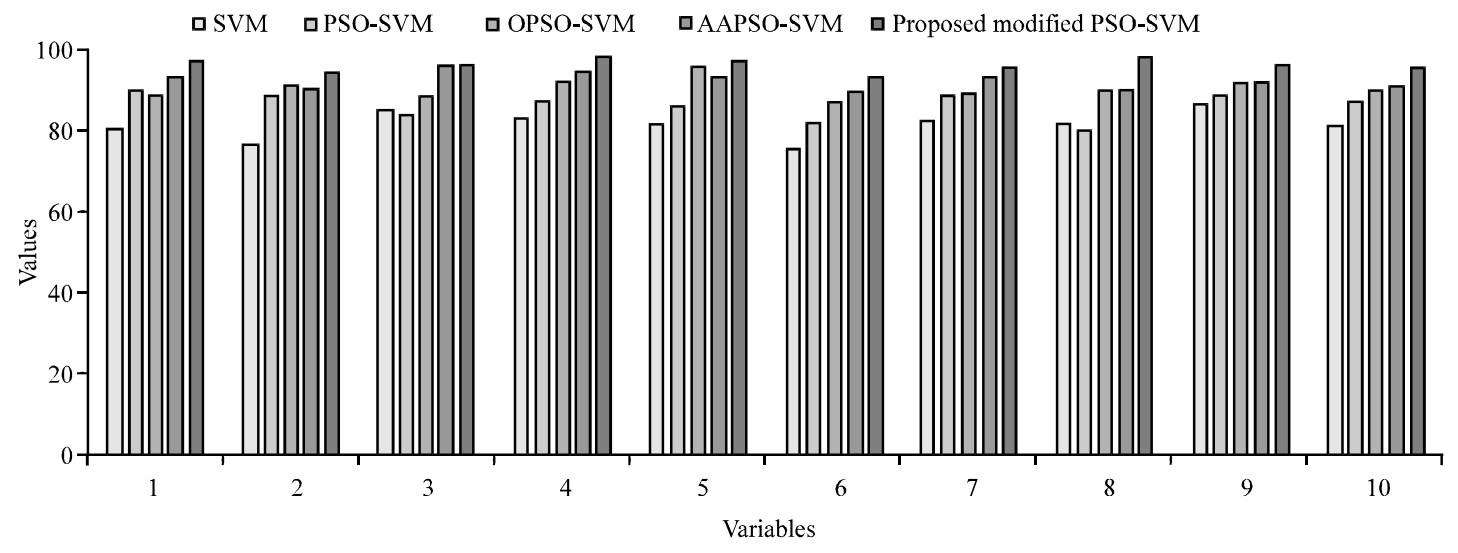

Fig. 5: Accuracy percentage

Table 1: The accuracy of the SVM, PSO-SVM, OPSO-SVM, AAPSOSVM and the proposed modified PSO-SVM

\begin{tabular}{|c|c|c|c|c|c|}
\hline Experiments & SVM & $\begin{array}{l}\text { PSO- } \\
\text { SVM }\end{array}$ & $\begin{array}{l}\text { OPSO- } \\
\text { SVM }\end{array}$ & $\begin{array}{l}\text { AAPSO- } \\
\text { SVM }\end{array}$ & $\begin{array}{l}\text { Proposed modified } \\
\text { PSO-SVM }\end{array}$ \\
\hline 1 & 80 & 90 & 89 & 93 & 97 \\
\hline 2 & 76 & 88 & 91 & 90 & 94 \\
\hline 3 & 85 & 84 & 88 & 96 & 96 \\
\hline 4 & 83 & 87 & 92 & 94 & 98 \\
\hline 5 & 81 & 86 & 95 & 93 & 97 \\
\hline 6 & 75 & 82 & 87 & 90 & 93 \\
\hline 7 & 82 & 89 & 89 & 93 & 95 \\
\hline 8 & 81 & 80 & 90 & 90 & 98 \\
\hline 9 & 86 & 89 & 92 & 92 & 96 \\
\hline 10 & 81 & 87 & 90 & 91 & 95 \\
\hline
\end{tabular}

Table 2: The sample of the optimal parameters for the SVM, PSO-SVM, OPSO-SVM, AAPSO-SVM and modified PSO-SVM on SCface datasets (first round only)

\begin{tabular}{lrc}
\hline Optimal parameters & $\mathrm{C}$ & $\sigma$ \\
\hline SVM & 112.10 & 0.44 \\
PSO-SVM & 89.23 & 0.65 \\
OPSO-SVM & 78.50 & 1.10 \\
AAPSO-SVM & 76.33 & 1.83 \\
Modified PSO-SVM & 81.10 & 0.32 \\
\hline
\end{tabular}

In addition, the optimal parameters of one round on the three datasets for the proposed modified PSO-SVM and the above-mentioned methods are demonstrated in Table 1 and 2 .

\section{CONCLUSION}

A new technique to optimize the parameters of support vector machine has been presented in this study. The proposed approach used the modified PSO method as a method to improve SVM parameters through modified PSO-SVM. Three human face datasets have been used to examine the overall performance of the proposed technique. The proposed modified PSO-SVM was compared in terms of accuracy with SVM and the recent methods like PSO-SVM, OPSO-SVM and AAPSO-SVM.
The proposed technique has gained 98\% accuracy among the other methods such as "SVM, PSO-SVM, OPSO-SVM and AAPSO-SVM". We will examine the proposed technique with the occlusion circumstance as a future work.

\section{ACKNOWLEDGEMENT}

The research would like to thank the Computer Science Department in Faculty of Education for Girls for their support of the research. Also, we would like to thank Assist Prof. Hasan farhan rashag for his advices.

\section{REFERENCES}

Abdulameer, M.H., D. Mohammed, S.A. Mohammed, M. Al-Azawi and Y.M.H. Al-Mayali et al., 2018. Face recognition technique based on Adaptive Opposition Particle Swarm Optimization (AOPSO) and Support Vector Machine (SVM). ARPN. J. Eng. Appl. Sci., 13: 2259-2266.

Abdulameer, M.H., S. Abdullah, S.N. Huda and Z.A. Othman, 2014. Support vector machine based on adaptive acceleration particle swarm optimization. Sci. World J., 2014: 1-8.

Abdullah, S.N.H.S., M.H. Abdulameer, N.A. Zamani, F. Rahim and K.A.Z. Ariffin et al., 2017. 2.5 D facial analysis via. bio-inspired active appearance model and support vector machine for forensic application. Intl. J. Adv. Comput. Sci. Appl., 8: 370-375.

Anonymous, 2007. CASIA face database. Chinese Academy of Sciences, Beijing, China.

Eberhart, R. and J. Kennedy, 1995. A new optimizer using particle swarm theory. Proceedings of the 6th IEEE International Symposium on Micro Machine Human Science, October 4-6, 1995, IEEE, Nagoya, Japan, ISBN:0-7803-2676-8, pp: 39-43. 
Grgic, M., K. Delac and S. Grgic, 2011. SCface-surveillance cameras face database. Multimedia Tools Appli., 51: 863-879.

Gross, R., I. Matthews, J.F. Cohn, T. Kanade and S. Baker, 2008. Multi-PIE. Proceedings of the IEEE 8th International Conference on Automatic Face and Gesture Recognition, September 17-19, 2008, IEEE, Amsterdam, Netherlands, ISBN:978-1-4244-2153-4, pp: 1-8.

Hasan, M., S.N.H.S. Abdullah and Z.A. Othman, 2013. Face recognition based on opposition particle swarm optimization and support vector machine. Proceedings of the 2013 IEEE International Conference on Signal and Image Processing Applications (ICSIPA'13), October 8-10, 2013, IEEE, Melaka, Malaysia, ISBN:978-1-4799-0267-5, pp: 417-424.

Hussein, H.A. and H.A.W. Habeeb, 2018. Parameter optimization of support vector machine using enhanced hybrid particle swarm optimization in non-linear face authintication problem. J. Eng. Appl. Sci., 13: 6162-6166.

Joachims, T., 1998. Text categorization with support vector machines: Learning with many relevant features. Lect. Notes Comput. Sci., 1398: 137-142.

Kennedy, J., 2011. Particle Swarm Optimization. In: Encyclopedia of Machine Learning, Sammut, C. and G.I. Webb (Eds.). Springer Science and Business Media, New York, USA., ISBN: 9780387307688, pp: 760-766.
Khadhraoui, T., S. Ktata, F. Benzarti and H. Amiri, 2016. Features selection based on modified PSO algorithm for $2 \mathrm{D}$ face recognition. Proceedings of the 2016 13th International Conference on Computer Graphics, Imaging and Visualization (CGiV), March 29-April 1, 2016, IEEE, Beni Mellal, Morocco, pp: 99-104.

Li, Y., K. Bontcheva and H. Cunningham, 2005. SVM Based Learning System for Information Extraction. In: Deterministic and Statistical Methods in Machine Learning, Winkler, J., N. Lawrence and M. Niranjan (Eds.). Springer, Berlin, Germany, ISBN: 978-3-540-29073-5, pp: 319-339.

Mosa, S.J., 2018. A critical comparative analysis for 2D and 2.5D face recognition techniques. J. Eng. Appl. Sci., 13: 8034-8042.

Raikwal, J.S. and K. Saxena, 2012. Performance evaluation of SVM and k-nearest neighbor algorithm over medical data set. Intl. J. Comput. Appl., 50: 35-39.

Wei, J., Z, Jian-Qi and Z. Xiang, 2011. Face recognition method based on support vector machine and particle swarm optimization. Expert Syst. Appl., 38: 4390-4393.

Yu, L., S. Wang, K.K. Lai and L. Zhou, 2008. Bio-inspired Credit Risk Analysis: Computational Intelligence with Support Vector Machines. Springer, Berlin, Germany, ISBN: 978-3-54077803-5, Pages: 241. 